\title{
Quality Assurance of Analytical Measurements- A Vital Element in Safety Performance in the Nuclear Field ${ }^{+}$
}

\author{
Elena Neacsu \\ Horia Hulubei National Institute for R\&D in Physics and Nuclear Engineering (IFIN-HH), 077125 Măgurele, \\ Romania; egneacsu@nipne.ro \\ † Presented at the Virtual Eurachem Workshop 2020- “Quality Assurance for Analytical Laboratories in the \\ University Curriculum", 14-15 July 2020; Available online: https://eurachem2020.ro/.
}

Published: 17 September 2020

\begin{abstract}
The (International Atomic Energy Agency) IAEA's fundamental safety objective is to protect people and the environment from harmful effects of ionizing radiation. The safety principles apply to all facilities and all activities to reduce existing radiation risks. Analytical quality assurance has gained in importance in many scientific areas, including the analysis of radioactive specimens that require a thorough investigation and regulations for safety and ecological reasons. The use of certified reference materials is an essential pillar for the assessment of the quality of analytical data. Still, such matrix-matched certified reference materials are unfortunately not available for most investigations relevant to the nuclear domain. Therefore, other strategies have to be established, i.e., to compare the analytical results obtained for a particular instrumental technique, with data from another methodology whose analyte detection is based on a different physical principle.
\end{abstract}

Keywords: quality assurance; analytical measurements; nuclear and radioactive materials; safety

\section{Introduction}

In the 20th century, analytical chemistry consisted mainly of gravimetry and titrimetry, and, without commenting or criticizing the achievements of the past, the students were not taught enough about measurement quality control (Q.C.) and assurance in the field of analytical chemistry. However, during the past 50 years, analytical chemistry has made a huge step forward due to the development of a complete series of measuring instruments for specific analytical techniques. In parallel, the need to produce analytical data of demonstrated quality was soon recognized, and an increased interest and effort lead to the issuance of a series of guidelines for analytical laboratories to provide more specific guidance to different measurement disciplines [1-3]. At present, numerous educational initiatives are taken for re-enforcing the analytical chemistry curriculum, in both conventional chemistry and measurement science. In that respect, students should be taught already early on in their curriculum about the importance of measurement uncertainty and accuracy of results.

Over the last decades, analytical quality assurance (Q.A.) has gained in importance in many scientific areas, including the analysis of radioactive specimens that require a thorough investigation and regulations for safety and ecological reasons. Intending to ensure the protection of people and the environment from harmful effects of ionizing radiation, the International Atomic Energy Agency developed and published Fundamental Safety Principles [4] that apply to facilities and activities that give rise to radiation risks. Taking into consideration that the safety principles are applicable 
throughout the entire lifetime of all facilities and all activities, there is an imperative need to perform the complete characterization of radioactive materials and to consider what level of uncertainty in the characterization information is acceptable. The uncertainty will relate to the extent of the characterization work and the accuracy and precision of results. Such characterization enables the quantities and categories of radioactive materials to be optimized for further planning purposes. It is important, therefore, that each measurement is conducted within the framework of a Q.A. program that, if followed, can be shown to achieve the desired objectives with the desired accuracy. In this paper are described the most critical activities in the nuclear field that require a thorough investigation for safety and ecological reasons and analytical techniques involved, specific uncertainty features of nuclear analytical techniques and several challenges in obtaining a better analytical performance.

\section{Nuclear/Radioactive Material Characterization}

The importance of composition control of the materials used in nuclear energy and applications of nuclear techniques in the industry, medicine, research, education is evident. The few important activities in the nuclear field that require a thorough investigation are presented below. It is not a detailed overview of the area, however, it illustrates the role of chemistry as one of the key factors for further nuclear energy development safely and economically, under safety principles. These activities have created anxiety about the radionuclides hazard, and Q.A. programs are part of the proofing that better knowledge is associated with lower radiation hazards.

In the nuclear industry, the knowledge of the composition and purity of fuels, moderators, coolants, and structural materials used in reactors is of essential importance [5-10]. The importance of the analytical characterization of nuclear materials is highlighted by very stringent requirements of nuclear fuel design. The nuclear fuel cycle is very complex, involving several stages, and different impurities can get incorporated into nuclear fuel, affecting its properties and performance. The major elemental composition of nuclear fuel is essential for the efficient and safe fission reaction in the fuel. Analogically, the design of other nuclear materials is required for their efficient performance. The amount of trace metallic as well as non-metallic elements present as impurities is needed to be determined to ensure the process optimization and avoid detrimental effects on the physical properties and integrity of the materials under the reactor operating and transient conditions. The operation of reactors and the reprocessing of partially spent nuclear fuels depend on the continuous control of chemical composition and sometimes pose real challenges to the analytical chemists as a consequence of the special conditions under which the analyses have to be performed.

Radioactive waste (R.W.) is a type of hazardous waste that contains radioactive material and represents a by-product of various nuclear technology processes. R.W. is hugely varied in terms of physical and chemical form, radioactivity, and the half-life of the radioactive elements contained, as well as volume, and requires treatment and management to successfully isolate it from interacting with the biosphere [11]. Tests and analyses to demonstrate that the waste meets the performance objectives established by the disposal acceptance criteria systematically with emphasis on Q.A. and Q.C., ways to strengthen confidence in decision-making processes. In this context, a whole range of both non-destructive and destructive measurement methods allowing access to the physical (density, volume, shape, the position of the waste and embedding matrixes, mechanical toughness, cracking, diffusion coefficient, gas release, thermal power, etc.), chemical (elemental composition, the content of toxic or reactive substances, etc.), and radiological characteristics (dose rate, alpha, beta, and gamma activity, isotopic composition and mass of nuclear materials, etc.) of nuclear waste or nuclear waste packages are used.

Nuclear forensics (N.F.) can be defined as "the examination of nuclear or other radioactive materials or evidence contaminated with radionuclides in the context of international or national law or nuclear security". Determination and accounting of nuclear materials are needed for the issues related to the health and safety of the workers and the public as well as for the prevention and the misuse of these materials for the proliferation of nuclear weapons. N.F. represents the support of nuclear security, and it is a critical element in preventing and detection of theft, sabotage, 
unauthorized access, illegal transfer, or other malicious acts involving nuclear material [12]. The term "nuclear forensic signatures" describes material characteristics such as chemical or isotopic composition, elemental concentrations, chemical impurities physical and chemical forms, physical dimensions, visual appearance and geometry and they may be used to link a material, either nuclear or radioactive, to individuals, locations, or processes. N.F. signature measurement involves analytical techniques from a wide range of disciplines, particularly analytical chemistry, radiochemistry (i.e., the chemistry of radioactive elements and compounds), and material science. Taking into consideration that N.F. support law enforcement or nuclear security investigations, such analysis should be performed under a quality assurance plan, validated analytical procedures, staff with demonstrated competencies, documented procedures, standard reporting forms, and records management.

Other practices from nuclear field that require characterization are preparation and use of radiopharmaceuticals, environmental radioactivity monitoring, radiological surveillance of foodstuffs, nuclear and radiological emergency preparedness, etc.

\section{Nuclear Analytical Measurements}

The term "nuclear analytical techniques" refers to techniques that provide the qualitative or quantitative determination of an element based on the characteristics and properties of the atomic nucleus. According to this definition, the following analytical techniques are considered as nuclear techniques: mass spectrometry, ion beam analysis, nuclear magnetic resonance spectrometry, Mössbauer spectrometry, neutron scattering, and diffraction, neutron activation analysis, isotopic dilution analysis, stable isotope, and radiotracer studies, and direct radioactivity determinations [13]. Some of the nuclear analytical techniques require appropriate irradiation, either by particles or high energy electromagnetic radiation. Therefore, their use is dependent on the availability of nuclear facilities (nuclear reactors, radioactive sources, accelerators, etc.). Nuclear analytical techniques are highly sensitive in the determination of the micro- and macro-amounts or trace elements in almost all matrix types.

Nuclear techniques have specific uncertainty features comparing with non-nuclear techniques, having an advantage because their error sources are traceable, but, at the same time, pointing to greater complexity and further work needed in particular cases [14]. A specific advantage appears in tracer measurement, where the best accuracy might be obtained by measuring either the ratio of the final to initial quantities of the tracer isotope or the ratio of the tracer to the targeted isotope. Nuclear analytical techniques are relative analytical techniques, and therefore require calibration using appropriate calibration standards, and the results should always be validated to be reliable and comparable. Consequently, the uncertainty component associated with the calibration will include an uncertainty contribution from the reference materials and an uncertainty contribution from the calibration line fitting or the scaling factor. Data acquisition in nuclear analytical techniques is based on the accumulation of counts resulting from a decay process from a higher energy level to a lower energy level by the emission of particles and/or radiation. A Poisson distribution characterizes these processes and, therefore, the uncertainty associated with those processes can be readily derived from the standard deviation of the Poisson distribution. Thus, is eliminated the need for sometimes tedious and lengthy repetitions of the measurements. For a sufficiently large number of counts, the normal distribution can be used as a good approximation of the Poisson distribution. Due to the radioactive decay, the measured quantities are not constant in time, and appropriate corrections for decay have to be applied. The correction factors for decay are typically exponential functions of a single or several decay constants and time. The decay constant is a measured physical quantity and, therefore, subject to uncertainty, and so is the measurement of time. The quantification of uncertainty will, therefore, frequently involve nonlinear components, whose contribution is not constant but is a function of the time intervals between the different steps in the analytical procedure. This characteristic feature of nuclear analytical techniques might introduce a lot of complexity in the quantification of uncertainty, but provides an opportunity for minimization of the uncertainty by an adequate scaling of the time intervals. The interaction of radiation, either 
particulate or electromagnetic, with matter is also a nonlinear process and, therefore, the efficiency of nuclear radiation detectors should be determined. In general, the uncertainty component associated with the response of a detection device in nuclear analytical techniques will be composed of a contribution from the standards used for the calibration, a contribution from the curve fitting process, and a contribution from the model used to describe the response function.

Radiation detection devices and associated electronics might show saturation effects and dead-time at high count rates induced by high-intensity radiation. The saturation and the dead-time effects are usually compensated, but the compensation will still introduce some uncertainty, which has to be evaluated. Nuclear analytical techniques involve irradiation devices to induce the activated or excited states in the target material. They are complex devices, usually equipped with protective shielding and remotely operated components. Due to this complexity, the reproducibility of the radiation field intensity, as well as its spectral and spatial distributions, is not readily achieved and will introduce other uncertainty components to be accounted for in the overall evaluation. Due to the nature of nuclear measurements, i.e., presence of background, and the fact that nuclear analytical techniques are often applied to measuring low activity samples, e.g., environmental radioactivity monitoring, the uncertainty quantification for the results close to detection limit requires special attention.

\section{Challenges in the Quality Assurance of Analytical Techniques in the Nuclear Field}

The use of certified reference materials (CRMs) is an essential pillar for the assessment of the quality of any acquired analytical data. CRMs provide the foundation for accurate and precise measurements that provide credible data, being a critical component in any Q.A. program, and necessary for developing, testing, and validating nuclear and radioanalytical methods and quantifying radionuclides. Such matrix-matched CRMs are unfortunately not available for most investigations relevant to the nuclear domain, and, unfortunately, nuclear CRMs present particular challenges for both the users and producers [15].

The lack of matrix-matched certified reference material can be overcome by comparing the analytical results obtained for a particular instrumental technique with data from another methodology whose analyte detection is based on a different physical principle. Additionally, the analysts can gain confidence in the robustness of their own methods by other means such as inter- or intra-laboratory comparison exercises or simulation models. Although analytical nuclear techniques and methods are well established, performing such tasks in the presence of radiation creates unique challenges and difficulties, and often requires specialized equipment and processing. One continual challenge is to maintain relevant expertise and capabilities due to the reduction of the number of young scientists entering the field.

\section{Conclusions}

Although it has been stated that the approach of reporting results of chemical measurements together with measurement uncertainty is relatively new, already 35 years ago that was policy for all measurements and an increased interest and effort led to the issuance of a series of guidelines for analytical laboratories to provide more specific guidance to different measurement disciplines. Additionally, numerous educational initiatives are taken for re-enforcing the analytical chemistry curriculum, and this both on the conventional chemistry and measurement science.

Over the last decades, analytical quality assurance (Q.A.) has gained in importance in many scientific areas, including the analysis of radioactive specimens that require a thorough investigation and regulations for safety and ecological reasons.

Many practices from the nuclear field such as nuclear/radioactive material characterization, management of radioactive waste, nuclear forensic, nuclear analytical measurements, preparation and use of radiopharmaceuticals, environmental radioactivity monitoring, radiological surveillance of foodstuffs, nuclear and radiological emergency preparedness require a wide range of modern instrument-based analytical techniques, specialized equipment and processing, and relevant expertise. 
The use of certified reference materials is an important pillar for the assessment of the quality of any acquired analytical data. Certified reference materials provide the foundation for accurate and precise measurements, but such matrix-matched certified reference materials are unfortunately not available for most investigations relevant to the nuclear domain. Extensive research for the development of advanced methods for physical and chemical analysis with increased sensitivity, reliability, and thereby enhanced accuracy is conducted to overcome present limitations.

\section{References}

1. IUPAC. Protocol for the Design, Conduct and Interpretation of Method Performance Studies. Pure Appl. Chem. 1995, 67, 331-343.

2. IUPAC. Harmonized Guidelines for Internal Quality Control in Analytical Chemistry Laboratories. Pure Appl. Chem. 1995, 67, 649-666.

3. EURACHEM. Quantifying Uncertainty in Analytical Measurement, 1st ed.; Laboratory of the Government Chemist Information Services: London, UK, 1995.

4. IAEA. Safety Standards, Fundamental Safety Principles; No. SF-1; IAEA: Viena, Austria, 2006.

5. Cross, J.N.; Kuhn, K.; Kunsberg, D.J.; Matonic, J.H.; Olson, A.C.; Rim, J.H.; Schake, A.R.; Wylie, E.M.; Tandon, L. Analytical chemistry of nuclear material: Case studies from Los Alamos National Laboratory, J. Radioanal. Nucl. Chem. 2018, 318, 1697-1712, doi:10.1007/s10967-018-6328-9.

6. Chmielewski, A.G. Chemistry for the nuclear energy of the future. Nukleonika 2011, 56, 241-249.

7. Sangita, D. Elemental Characterization of Nuclear Materials using Total reflection X-Ray Fluorescence Spectrometry, Trends. Anal. Chem. 2019, 116, doi:10.1016/j.trac.2019.04.017.

8. Complexity in nuclear materials. Nat. Mater. 2015, 14, 245, doi:10.1038/nmat4234.

9. Ramaniah, M.V. Analytical chemistry of fast reactors fuel-A review. Pure Appl. Chem. 1982, 54, 889-908.

10. Rodriquez, M.; Demmer, R.; Berg, J.; McGrath, C.; Brough, D.; Thompson, S. Nuclear Materials Characterization in the Materials and Fuels Complex Analytical Hot Cells. In Proceedings of the WM2009 Conference, Phoenix, AZ, USA, 1-5 March 2009.

11. IAEA. TECDOC-1537. Strategy and Methodology for Radioactive Waste Characterization; IAEA: Vienna, Austria, 2007.

12. Mayer, K.; Wallenius, M.; Lützenkirchen, K.; Galy, J.; Varga, Z.; Erdmann, N.; Buda, R.; Kratz, J.V.; Trautmann, N.; Fifield, K. Nuclear Forensics: A Methodology Applicable to Nuclear Security and to Non Proliferation. J. Phys. Conf. Ser. 2011, 312, 062003, doi:10.1088/1742-6596/312/6/062003.

13. de Goeij, J.J.; Bode, P. Nuclear Analytical Techniques: Strong and Weak Points, Threats and Opportunities. In Proceedings of the International Symposium on Harmonisation of Health Related Environmental Measurements Using Nuclear and Isotopic Techniques, Hyderabad, India, 4-7 November 1996.

14. IAEA. IAEA-TECDOC-1401. Quantifying Uncertainty in Nuclear Analytical Measurements; IAEA: Viena, Austria, 2004.

15. Inn, K.G.W.; Johnson, C.M., Jr.; Oldham, W.; Jerome, S.; Tandon, L.; Schaaff, T.; Jones, R.; Mackney, D.; Griggs, J.; MacKill, P.; et al. The urgent requirement for new radioanalytical certified reference materials for nuclear safeguards, forensics and consequence management. J. Radioanal. Nucl. Chem. 2013, 296, 5-22. 